\title{
AVALIAÇÃO DOS NÍVEIS DE RUÍDO E VIBRAÇÃO DE UM CONJUNTO TRATOR-PULVERIZADOR, EM FUNÇÃO DA VELOCIDADE DE TRABALHO
}

Larissa Nunes dos Santos ${ }^{1}$, Haroldo Carlos Fernandes ${ }^{2}$, Amaury Paulo de Souza ${ }^{3}$, Marconi Ribeiro Furtado Júnior Remo Macieira Figueiredo Silva ${ }^{5}$

\section{RESUMO}

Objetivou-se com este trabalho, avaliar os níveis de ruído e vibração de um conjunto mecanizado trator-pulverizador, em função da velocidade de trabalho. O trabalho foi conduzido no Laboratório de Mecanização Agrícola, do Departamento de Engenharia Agrícola da Universidade Federal de Viçosa - MG. Para determinação dos níveis de ruído e vibração transmitida ao corpo inteiro do operador foram utilizados um dosímetro modelo Wed007, fabricado pela $01 \mathrm{~dB}$ do Brasil e um acelerômetro triaxial modelo Maestro respectivamente. As velocidades de trabalho utilizadas foram de 4,0; 5,0; e 7,0 $\mathrm{km} \mathrm{h}^{-1}$. Os resultados obtidos para os níveis de ruído foram 98,2 $\mathrm{dB}$ (A) para a velocidade de 4,0 $\mathrm{km} \mathrm{h}^{-1}, 98,1 \mathrm{~dB}(\mathrm{~A})$ para 5,0 $\mathrm{km} \mathrm{h}^{-1} \mathrm{e} 97,4 \mathrm{~dB}(\mathrm{~A})$ para 7,0 $\mathrm{km} \mathrm{h}^{-1}$. Na análise da vibração os resultados encontrados foram $0,58\left(4,0 \mathrm{~km} \mathrm{~h}^{-1}\right) ; 0,60\left(5,0 \mathrm{~km} \mathrm{~h}^{-1}\right)$; e $0,60\left(7,0 \mathrm{~km} \mathrm{~h}^{-1}\right) \mathrm{m} \mathrm{s}^{-2}$. De acordo com os resultados, concluiu-se que todos os níveis de ruído encontrados foram superiores ao estabelecido pela norma NR-15 de $85 \mathrm{~dB}(\mathrm{~A})$ para uma jornada de trabalho de 8 horas por dia, sendo que o aumento da velocidade culminou na redução dos níveis de ruído. A vibração do trator-pulverizador obteve valores acima dos definidos pelos limiares da Diretiva europeia de $0,5 \mathrm{~m} \mathrm{~s}^{-2} \mathrm{em}_{\text {todas }}$ velocidades avaliadas, também sendo influenciada pela velocidade de deslocamento do conjunto mecanizado.

Palavras-chave: ergonomia, mecanização agrícola, pulverizador de barra

\section{ABSTRACT \\ EVALUATION OF LEVELS OF NOISE AND VIBRATION OF A TRACTOR-SPRAY SET, FOR EACH WORKING SPEED}

The objective of this study was to evaluate the noise and vibration levels of a mechanized tractor-sprayer set, in function of the working speed. The work was conducted in the Laboratory of Agricultural Mechanization, Department of Agricultural Engineering, Universidade Federal de Viçosa - MG. To determine the levels of noise and vibration transmitted to the body of the operator a dosimeter model Wed007 was used, manufactured by $01 \mathrm{~dB}$ in Brazil and one triaxial accelerometer model Maestro, respectively. The working speeds used were 4.0, 5.0 and $7.0 \mathrm{~km} \mathrm{~h}^{-1}$. The results obtained for the noise levels were $98.2 \mathrm{~dB}$ (A) for the speed of $4.0 \mathrm{~km} \mathrm{~h}^{-1}, 98.1 \mathrm{~dB}$ (A) for $5.0 \mathrm{~km} \mathrm{~h}^{-1}$ and $97.4 \mathrm{~dB}(\mathrm{~A})$ for $7.0 \mathrm{~km} \mathrm{~h}^{-1}$. In the vibration analysis the results were $0.58\left(4.0 \mathrm{~km} \mathrm{~h}^{-1}\right) ; 0.60\left(5.0 \mathrm{~km} \mathrm{~h}^{-1}\right)$; and $0.60\left(7.0 \mathrm{~km} \mathrm{~h}^{-1}\right) \mathrm{m} \mathrm{s}^{-2}$. According to the results it was concluded that all noise levels were superior to the established standard NR-15 of 85 $\mathrm{dB}$ (A) for a work shift of 8 hours per day, and increased speed resulted in reduction noise levels. The vibration of the tractor-sprayer showed values above the thresholds set by the European Directive of $0.5 \mathrm{~m} \mathrm{~s}^{-2}$ at all speeds evaluated, also being influenced by the forward speed of the mechanized set.

Keywords: ergonomics, agricultural mechanization, boom sprayer.

\section{Recebido para publicação em 26/08/2013. Aprovado em 23/09/2013.}

\footnotetext{
1 - Engenheira Florestal, Mestranda em Engenharia Agrícola, UFV/Viçosa-MG, larissa.nunes@ufv.br

2 - Engenheiro Agrícola, Professor Associado da UFV/Viçosa-MG, haroldo@ufv.br

3 - Engenheiro Florestal, Professor Titular da UFV/Viçosa-MG, amaurysouza@ufv.br

4 - Engenheiro Agrônomo, Doutorando em Engenharia Agrícola, UFV/Viçosa-MG

5 - Engenheiro Florestal, Mestrando em Engenharia Agrícola, UFV/Viçosa-MG, remo.silva@ufv.br
}

\section{REVENG}




\section{INTRODUÇÃO}

$\mathrm{O}$ trator tem a função tracionar, acionar e realizar o levante dos implementos utilizados em todas as operações de produção agrícola, tais como: preparo do solo, plantio, cultivo e colheita, substituindo as máquinas rudimentares que possuem como principal fonte de potência a força animal, tornando-as menos árdua e mais eficiente.

O tamanho das áreas de produção e quantidade de produtores no país está em crescimento constante tendo como resposta o aumento na utilização de fitossanitários utilizados para eliminação de plantas daninhas, fungos, entre outros. O conjunto mecanizado utilizado nesta operação pose ser composto por pulverizadores hidráulicos, pneumáticos ou hidropneumático acoplados ao sistema de levante hidráulico e/ou barra de tração acionados pela tomada de potência do trator.

Atualmente, os pulverizadores mais utilizados são os hidráulicos, visto sua facilidade de uso. Entretanto, a operação com tal máquina pode ocasionar uma série de danos à saúde de seus operadores, sendo necessária a caracterização de parâmetros ergonômicos (vibrações e ruídos, por exemplo) para avaliar se os mesmos estão dentro dos limites aceitáveis pelas normas vigentes.

No Ministério do Trabalho, a norma que resguarda $\mathrm{o}$ trabalhador, no que diz respeito às atividades $\mathrm{e}$ operações insalubres é a NR-15, a qual também estabelece limites de exposição ao ruído durante toda sua jornada de trabalho. Como não existem normas no Brasil que se referem a limites de exposição à vibração, as normas internacionais tem sido largamente utilizadas, dentre elas tem-se a ISO 21361 (1977), e a diretiva 2002/44/CE da Comunidade Europeia que estipulam níveis de ação e limites de exposição humana a vibrações do corpo inteiro.

A ergonomia pode ser definida como o estudo da interação dos seres humanos ao seu ambiente de trabalho, visando melhorias de bem estar, conforto e segurança, através da aplicação de teorias e métodos a projetos operacionais (ABERGO, 2012).

A redução da eficiência da operação e aumento de riscos a saúde do trabalhador é resultado da deficiência do projeto da máquina, do tempo de exposição diário, da falta de treinamento e experiência do operador e dos procedimentos e posturas adotadas para realização do trabalho. Machado et al. (2002) afirmam que é impossível obter produtividade satisfatória se a operação não suprir alguns requisitos básicos para o operador.

Apesar da atual importância no cenário agrícola e florestal, boa parte dos tratores produzidos no país não são projetados para atender a relação homem-máquina (adequação do trabalho ao trabalhador), sendo a concepção do projeto voltada para a otimização dos parâmetros operacionais e desempenho, obrigando muitas vezes o operador a trabalhar exposto às condições climáticas, ao ruído e a vibração.

Baesso et al. (2008) encontraram níveis de ruído próximo ao ouvido direito do operador durante a operação de pulverização tratorizada com assistência de ar ligada na barra de 104,85 $\mathrm{dB}(\mathrm{A})$, alertando o risco de hipoacusia na execução intensiva desta atividade.

Altos níveis de ruído podem ocasionar a redução e/ou até mesmo a perda de audição do trabalhador, sendo fonte de desconforto e redução do rendimento do conjunto homem-máquina.

Outro fator de grande relevância para redução dos riscos a saúde do operador é a vibração. Segundo Lanças et al. (2009), a vibração exerce influência direta no afastamento por doença do trabalho no país. A transmissão de vibração para o ser humano resulta em desconforto e aumento da fadiga do trabalhador podendo ser classificada de acordo com a região do corpo atingida em: Vibrações de Corpo inteiro (atividades de transporte, tais como caminhão, trator, empilhadeira, ônibus) e Vibrações de Extremidades (principalmente mãos, braços). A exposição às vibrações de corpo inteiro pode ocasionar em patologias na região lombar e lesões da coluna vertebral (SOEIRO, 2011).

Em um estudo variando a potência do trator; o tipo de pista de deslocamento, pressão interna dos pneus e velocidade média de deslocamento, Lanças et al. (2009), puderam observar que o aumento da velocidade de deslocamento do trator culmina no aumento da vibração sofrida pelo operador, sendo necessário diminuir o tempo máximo de exposição.

Com base nesse contexto, objetivou-se com este trabalho, avaliar os níveis de ruído e vibração no conjunto mecanizado trator-pulverizador, em função da velocidade de trabalho.

\section{MATERIAL E MÉTODOS}

O experimento foi conduzido no mês de março 2013, no Laboratório de Mecanização Agrícola do Departamento de Engenharia Agrícola da Universidade Federal de Viçosa - MG.

No momento da coleta de dados a temperatura ambiente era de $31,2^{\circ} \mathrm{C}$ e a velocidade do vento $1,6 \mathrm{~m} \mathrm{~s}^{-1}$. O referido trabalho foi conduzido sobre 
um solo arado.

O experimento foi estabelecido em delineamento inteiramente casualizado (DIC), com 4 repetições. Cada parcela dispôs de uma área de $25 \mathrm{~m}$ de comprimento, deixando-se $5 \mathrm{~m}$ para estabilização do conjunto mecanizado.

O conjunto mecanizado utilizado foi composto de um trator agrícola John Deere ${ }^{\circledR}$ (Figura 1), modelo 5705, 4x2 com tração dianteira auxiliar (TDA) e potência de $62,56 \mathrm{~kW}(85 \mathrm{cv})$ no motor a $2400 \mathrm{rpm}$, equipado com pneus diagonais, e um pulverizador Falcon Vortex, marca Jacto, com tanque de 600 L e ventilador para uso de assistência de ar na barra de pulverização, acoplado ao trator nos três pontos do sistema hidráulico e acionado pela tomada de potência (Figura 2).

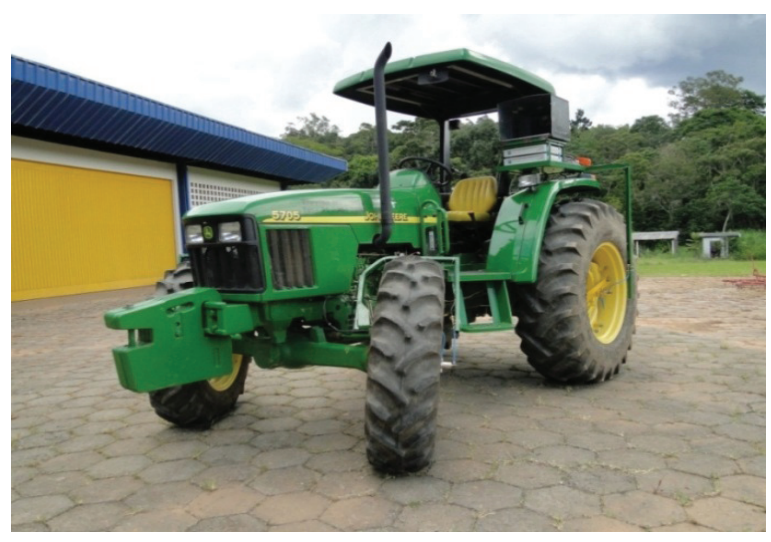

Figura 1. Trator John Deere, modelo 5705 utilizado no experimento.

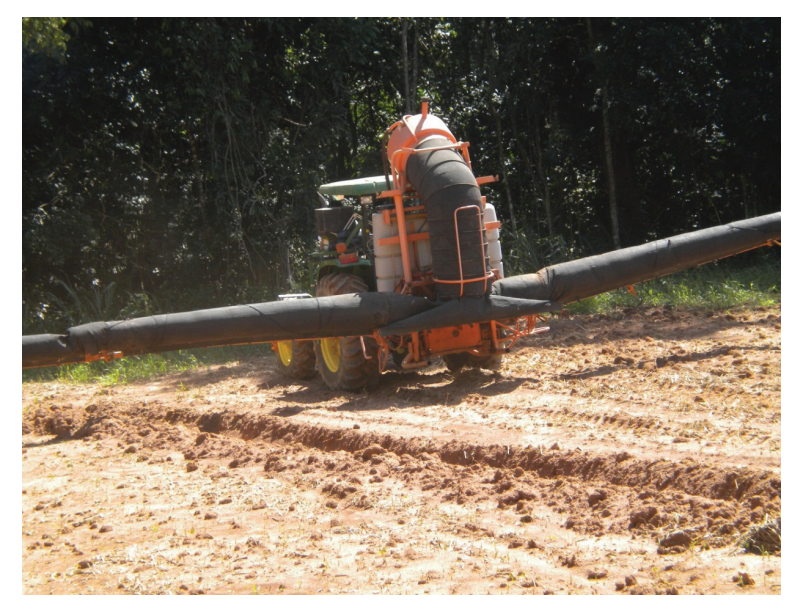

Figura 2. Conjunto trator-pulverizador utilizado no experimento.

O solo utilizado foi caracterizado como Latossolo Vermelho-Amarelo distrófico. As velocidades foram de 4,0 ( $1^{\mathrm{a}}$ reduzida $) ; 5,0\left(1^{\mathrm{a}}\right.$ intermediária); e 7,0 (2 $2^{\mathrm{a}}$ intermediária) $\mathrm{Km} \mathrm{h}^{-1}$, com a rotação de $540 \mathrm{rpm}$ na tomada de potência.

Os níveis de ruído foram detectados por meio do uso de um dosímetro modelo Wed007, fabricado pela $01 \mathrm{~dB}$ do Brasil (Figura 3), operando no circuito de compensação "A", o qual origina níveis de pressão sonora ponderadas em "A". Este aparelho é responsável pela avaliação da dose de ruído absorvida durante a exposição de ruído pelo operador em sua jornada de trabalho.

A escala utilizada para leituras foi o decibel (dB). As medições dos níveis de ruído foram realizadas com o aparelho posicionado à altura do ouvido esquerdo do operador (Figura 4) por ser o lado onde se encontra o escapamento do trator.

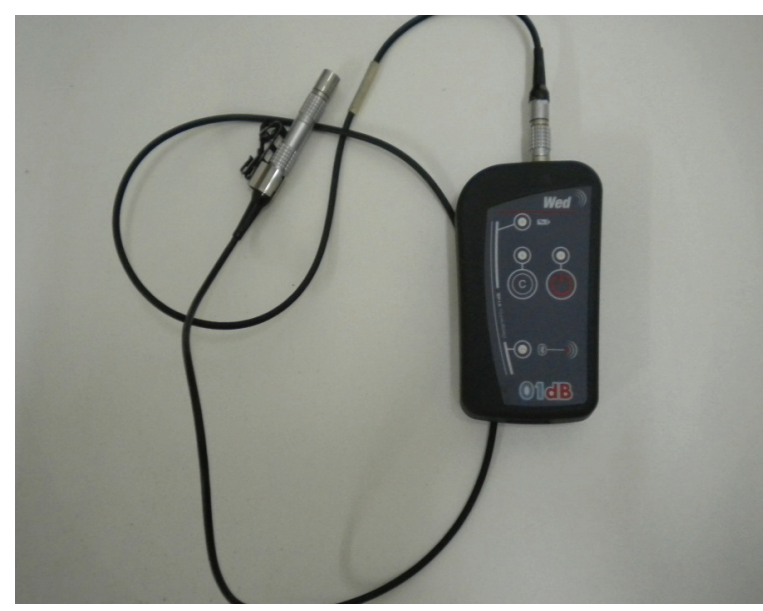

Figura 3. Dosímetro utilizado no experimento.

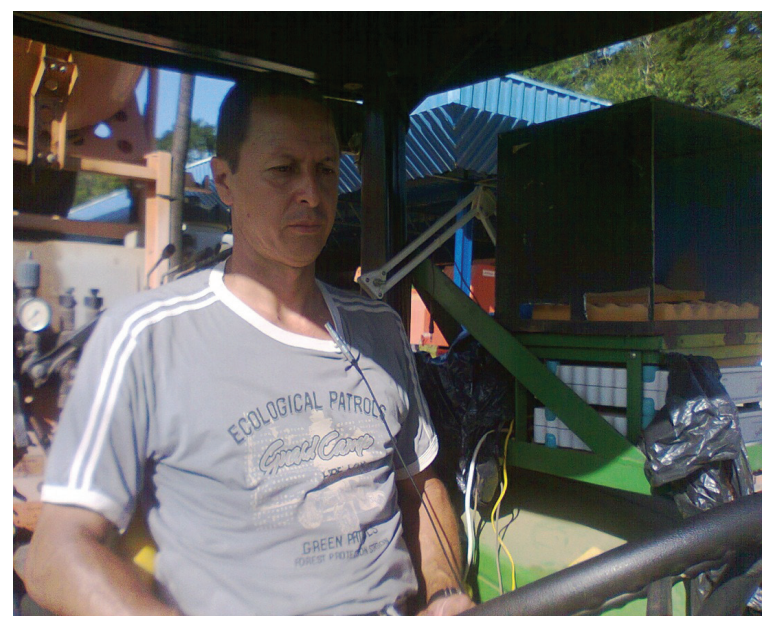

Figura 4. Posicionamento do microfone do dosímetro durante a coleta de dados.

O tempo de exposição diário do operador foi avaliado de acordo com os níveis de ruído determinados pela Norma regulamentadora $n^{\circ} 15$ - Atividades e Operações Insalubres (MTE, 1978) (Quadro 1).

\section{REVENG \\ 112-118p.}

ENGENHARIA NA AGRICULTURA, VIÇOSA - MG, V.22 N.2, MARÇO / ABRIL 2014 
Quadro 1. Limites de Exposição ao Ruído, sem uso de Equipamento de Proteção Individual (EPI's).

\begin{tabular}{cc}
\hline Nível de Ruído (dBA) & Máxima Exposição Diária Permissível \\
\hline 85 & 8 horas \\
86 & 7 horas \\
87 & 6 horas \\
88 & 5 horas \\
89 & 4 horas e 30 minutos \\
90 & 4 horas \\
91 & 3 horas e 30 minutos \\
92 & 3 horas \\
93 & 2 horas e 40 minutos \\
94 & 2 horas e 15 minutos \\
95 & 2 horas \\
96 & 1 hora e 45 minutos \\
98 & 1 hora e 15 minutos \\
100 & 1 hora \\
102 & 45 minutos \\
104 & 35 minutos \\
105 & 30 minutos \\
106 & 25 minutos \\
108 & 20 minutos \\
110 & 15 minutos \\
112 & 10 minutos \\
114 & 8 minutos \\
115 & 7 minutos \\
&
\end{tabular}

Para medição da vibração transmitida ao corpo inteiro foi utilizado um acelerômetro triaxial modelo Maestro (Figura 5), localizado e fixado no assento do trator, efetuando leituras nos três eixos (x, y e z) conforme a Figura 6.

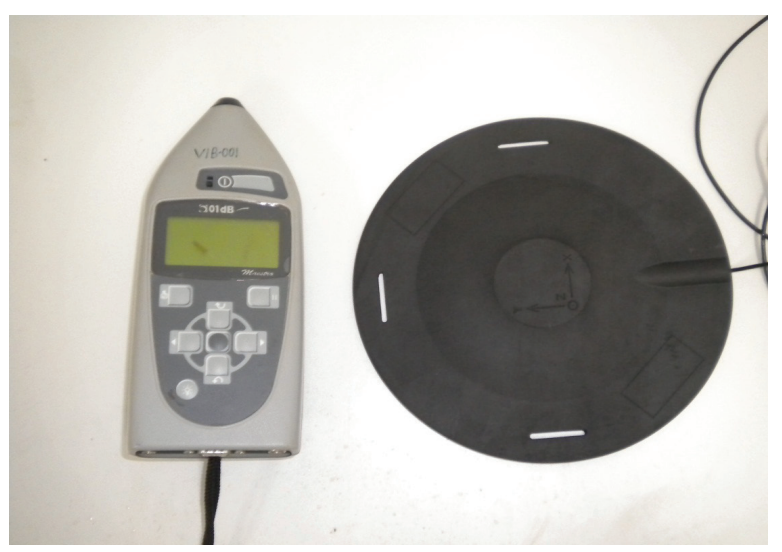

Figura 5. Acelerômetro triaxial utilizado no experimento.

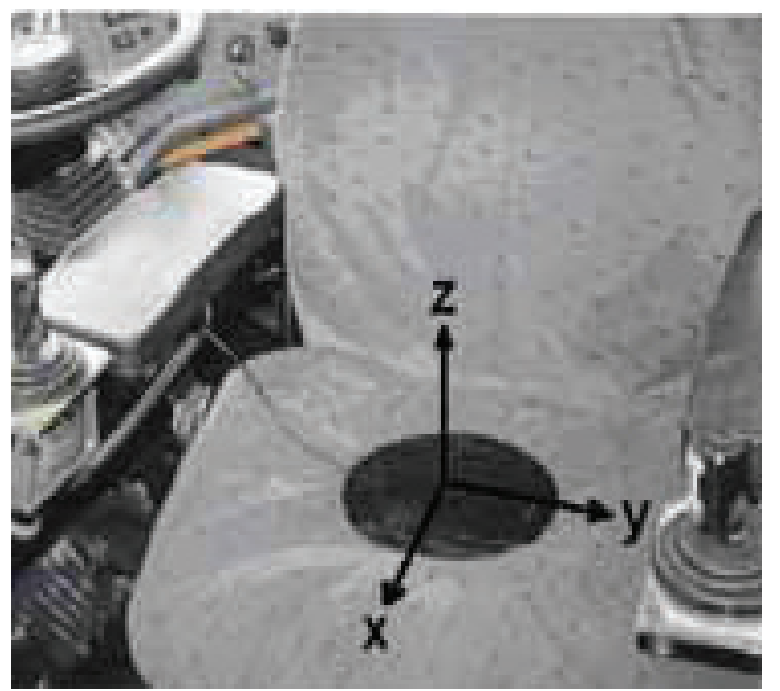

Figura 6. Vista do acelerômetro utilizado no assento e dos eixos de medição, posicionados para avaliação da exposição de condutor de veículo. Fonte: NHO01 
As medições do tempo de exposição do operador à vibração foram efetuadas de acordo com a norma internacional ISO 2136-1 (1977). A avaliação do tempo de exposição do operador à vibração foi comparada com base no definido pela International Organization for Standardization ISO 2136-1 (1977) e pela diretiva 2002/44/CE da Comunidade Europeia.

Os dados de vibração $\left(\mathrm{m} \mathrm{s}^{-2}\right)$ obtidos foram submetidos à análise de variância e posteriormente analisados por meio de regressão linear.

Para a realização do procedimento estatístico citado anteriormente foi utilizado o programa computacional ASSISTAT versão 7.6 beta e o Microsoft Excel versão 2010.

\section{RESULTADOS E DISCUSSÃO}

Os valores obtidos para os níveis médio representativos da exposição do trabalhador ao ruído foram acima do que os estabelecidos pela norma NR-15 de $85 \mathrm{~dB}(\mathrm{~A})$ para uma jornada de trabalho de 8 horas por dia, sendo necessário o uso de protetor auricular para proteção e prevenção de problemas a saúde do trabalhador.

No Quadro 2 apresentam-se os limites de exposição ao ruído, sem o uso de EPI's, para a atividade de pulverização no solo em diferentes velocidades de trabalho de acordo com a NR-15.

Observa-se na Figura 7 que com redução na velocidade de trabalho ocorreu aumento no nível de ruído. Isso provavelmente ocorreu em função das menores velocidades estarem nas marchas com maior torque, as quais emitem maior ruído, uma vez que a rotação do motor permaneceu constante, simulando a condição de operação normal do operador. Segundo Zoppello et al. (1995), os fatores que exercem maior influência nos níveis de ruído são a rotação e a vibração dos órgãos internos transmissores de potência da máquina.

Alvarenga (2009) obteve resultados semelhantes para pulverizadores hidráulicos de barra acoplados a tratores sem cabine em um estudo comparando níveis de ruído com tratores com e sem cabine. Souza et al. (2004), avaliando níveis de ruídos emitidos por uma recolhedora-trilhadora de feijão, também observaram maiores níveis de ruído em menores velocidades de trabalho.

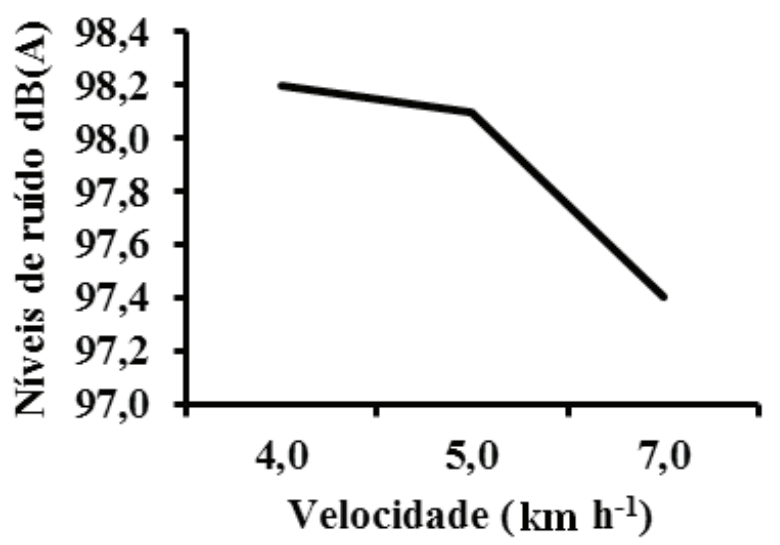

Figura 7. Nível médio representativo da exposição do trabalhador ao ruído em função da velocidade de trabalho.

Os dados obtidos em relação aos níveis de vibração não apresentaram variações bruscas com a mudança da velocidade. No entanto, estes valores se apresentaram acima do limite de ação de 0,5 $\mathrm{m} \mathrm{s}^{-2}$ estabelecido pela diretiva Europeia, sendo necessário avaliar as condições de amortecimento no assento do trator para realização desta atividade.

O comportamento da vibração $\left(\mathrm{m} \mathrm{s}^{-2}\right)$ de acordo com o aumento da velocidade seguiu conforme apresentado na Figura 8. Observa-se que

Quadro 2. Limites de Exposição ao Ruído, sem uso de Equipamento de Proteção Individual (EPI's) conforme a NR-15

\begin{tabular}{ccc}
\hline Velocidade & Ruído $\mathrm{dB}(\mathrm{A})$ & Máxima Exposição Diária Permissível \\
\hline $4,0 \mathrm{Km} \mathrm{h}^{-1}$ & 98,2 & 1 hora e 15 minutos \\
$5,0 \mathrm{Km} \mathrm{h}^{-1}$ & 98,1 & 1 hora e 15 minutos \\
$7,0 \mathrm{Km} \mathrm{h}^{-1}$ & 97,4 & 1 hora e 22 minutos \\
\hline
\end{tabular}


Quadro 3. Efeito da intensidade de vibração para o conforto do operador conforme a ISO 2136-1 (1997).

\begin{tabular}{ccc}
\hline Velocidade & Vibração $\left(\mathrm{m} \mathrm{s}^{-2}\right)$ & Reações com relação ao conforto \\
\hline $4,0 \mathrm{~km} \mathrm{~h}^{-1}$ & 0,4925 & Pouco desconfortável \\
$5,0 \mathrm{~km} \mathrm{~h}^{-1}$ & 0,5050 & Bastante desconfortável \\
$7,0 \mathrm{~km} \mathrm{~h}^{-1}$ & 0,5200 & Bastante desconfortável \\
\hline
\end{tabular}

o aumento da velocidade fez com que os valores de vibração aumentassem quase que de maneira constante. Isso pode ter acontecido pelas condições as quais se encontravam o solo (arado, sem pedras e tocos), por não haver mudanças na rotação do motor, reduzindo a vibração dos órgãos internos da máquina, e devido às velocidades escolhidas propiciarem estabilidade à barra de pulverização. Provavelmente em maiores velocidades haveria um menor equilíbrio da barra, em consequência principalmente do vento, resultando numa maior vibração do conjunto mecanizado.

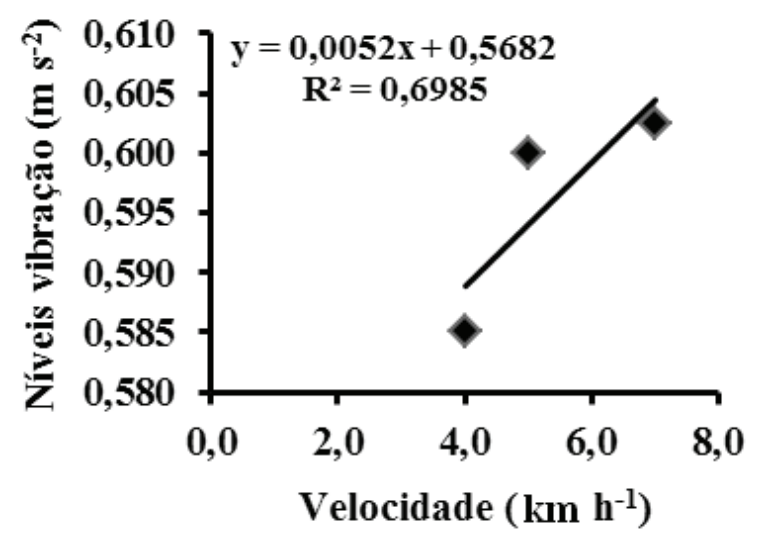

Figura 8. Nível de vibração $\left(\mathrm{m} \mathrm{s}^{-2}\right)$ no solo para exposição diária de 8 horas, em função da velocidade de trabalho.

Roth (2010), ao avaliar as vibrações atuantes na interface assento-operador, em um trator agrícola, obteve aumento nos valores de aceleração com o aumento da velocidade em diferentes tipos de terreno.

No Quadro 3 estão representados os valores totais da vibração de aceleração ponderada RMS $\left(\mathrm{m} \mathrm{s}^{-2}\right)$ e os efeitos de sua intensidade no conforto do operador de acordo com ISO 2136-1 (1997).

Pode-se perceber que o conforto do operador era maior quando o trator operava em menor velocidade. Isso pode ter ocorrido em consequência do solo oferecer menor estabilidade, por este ser irregular, aumentando a vibração com o aumento da velocidade. No entanto, a diferença entre os valores encontrados foi mínima, sendo possível reduzir o desconforto do operador através de pequenas melhorias na suspensão, molas e assento do trator.

Segundo Roth (2010), o conforto ou desconforto do assento das máquinas tem sido motivo de preocupação para os operadores, uma vez que eles passam longos períodos de tempo sentados.

\section{CONCLUSÃO}

- Os níveis de ruído foram superiores ao estabelecido pela norma NR-15 de $85 \mathrm{~dB}(\mathrm{~A})$ para uma jornada de trabalho de 8 horas por dia, em todas as condições avaliadas;

- O aumento da velocidade culminou na redução dos níveis de ruído;

- Os valores da vibração do trator-pulverizador atingiram valores acima dos definidos pelos limiares da diretiva europeia de $0,5 \mathrm{~m} \mathrm{~s}^{-2} \mathrm{em}$ todas as velocidades estudadas;

- A velocidade de deslocamento do conjunto mecanizado influenciou o comportamento da vibração.

\section{REFERÊNCIAS BIBLIOGRÁFICAS}

ABERGO. Associação Brasileira de Ergonomia. Apresenta o conceito de ergonomia. Disponível em: $<\quad$ http://www.abergo.org.br/internas.php?pg=O que_e_ergonomia $>$. Acesso em 04 de mar. 2013.

ALVARENGA, C.B. Avaliação de pulverizadores hidráulicos de barra na região de Uberlândia - MG. 2009. 71f. Dissertação (Mestrado em 
Agronomia - Fitotecnia) - Universidade Federal de Uberlândia, Uberlândia, 2009.

BAESSO, M.M.; TEIXEIRA M.M.; RODRIGUES JUNIOR, F.A.; MAGNO JUNIOR, R.G.; FERNANDES, H.C. Avaliação do nível de ruído emitido por um conjunto trator-pulverizador com e sem assistência de ar. Engenharia na Agricultura, Viçosa, MG, v.16, n.4, p.400-407, out/dez, 2008.

DIRETIVA 2002/44/CE. Relativa às prescrições mínimas de segurança e saúde respeitantes à exposição dos trabalhadores aos riscos devidos aos agentes físicos (vibrações). Parlamento Europeu e do Conselho, 2002.

ISO 2631-1. "Mechanical vibration and shock - evaluation of human exposure to wholebody vibration - Part I: general requirements". Switzerland: International Standard, 1997.

LANÇAS, K.P.; TOSIN, R.C.; ARAUJO, J.A.B.; MONTEIRO, L.A.; GUERRA, S.P.S.. Avaliação da Vibração Ocupacional no Corpo Inteiro para Tratores Agrícolas de 55,2 kw e 109,5 kw. CONBEA, Juazeiro, BA/ Petrolina, PE, ago. 2009.

MACHADO, C.C.; SILVA, E.N.; PEREIRA, R.S. O Setor Florestal Brasileiro e a Colheita Florestal. In: Machado, C.C. (Ed.) Colheita florestal. 2ed. Viçosa: UFV, 2002, p.15-42.
MTE. - Ministério do Trabalho e Emprego. Atividades e operações insalubres: NR 15. Portaria MTb n. ${ }^{\circ}$ 3.214, de 08 de junho de 1978. Disponível em: <http://portal.mte.gov.br/data/fi les/8A7C816A36A27C140136A8089B344C39/ NR-15\%20(atualizada\%202011)\%20II.pdf $>$, Acesso em: 15 de fevereiro de 2013.

ROTH, C.W. Transmissibilidade da vibração e distribuição da pressão na interface assentooperador de tratores agrícolas em condições dinâmicas. 2010. 142 f. Tese (Doutorado em Engenharia Agrícola - Mecanização Agrícola) - Universidade Federal de Santa Maria, Santa Maria, 2010.

SOEIRO, N.S. Vibrações e o corpo humano: Uma avaliação ocupacional. I Workshop de Vibração e Acústica da Região Norte, Tucuruí, Pará, 2011.

SOUZA, L.H.; VIEIRA, L.B.; FERNANDES, H.C.; LIMA, J.S.S. Níveis de ruído emitidos por uma recolhedora-trilhadora de feijão. Revista Engenharia Agrícola, Viçosa, MG, v.24, n.3, p.745-749, abr./jun., 2004.

ZOPPELLO, G., MONARCA, D., CECCHINI, M. et al. Aziende agricole, il rischio da rumore. Macchine e Motori Agricoli, v.2, n.10, p.9-16, 1995. 